Comment. Math. Helv. 72 (1997) 101-109

(C) 1997 Birkhäuser Verlag, Basel

0010-2571/97/010101-09 \$1.50+0.20/0

Commentarii Mathematici Helvetici

\title{
Essential cohomology of finite groups
}

\author{
Alejandro Adem* and Dikran Karagueuzian**
}

\begin{abstract}
In this paper we prove that a finite group $G$ with Cohen-Macaulay mod $p$ cohomology will have non-trivial undetectable elements in $H^{*}\left(G, \mathbb{F}_{p}\right)$ if and only if $G$ is a $p$-group such that every element of order $p$ in $G$ is central. Applications and examples are also provided.
\end{abstract}

Mathematics Subject Classification (1991). $20 \mathrm{~J} 06$.

Keywords. Cohomology of finite groups.

\section{$\S 0$ Introduction}

One of the main difficulties in calculating or understanding the mod $p$ cohomology of a finite group $G$ is the fact that in many instances non-trivial cohomology classes will appear that restrict trivially on all proper subgroups. Such "essential" cohomology (denoted $\operatorname{Ess}^{*}(G)$ ) is difficult to obtain and yet seems to be an intrinsic contribution of some importance. On the other hand, it is evident from the definition that such groups will be "universal detectors" in the cohomology of finite groups. However, this detection scheme is much too general to be of any practical significance. Rather, one would hope that a certain accessible and well-understood collection of these groups would suffice under favorable conditions. In particular a group-theoretic characterization of such groups would be highly desirable.

In this paper we obtain such a result for certain groups, namely those whose cohomology ring is Cohen-Macaulay (i.e. free and finitely generated over a polynomial subalgebra). More precisely, we prove the following

Theorem 2.1. Let $G$ be a finite group, then the following two conditions are equivalent:

(1) $H^{*}\left(G, \mathbb{F}_{p}\right)$ is Cohen-Macaulay and contains non-trivial essential elements

(2) $G$ is a p-group and every element of order $p$ in $G$ is central.

\footnotetext{
*Partially supported by an NSF grant.

** Supported by an NSF Postdoctoral Fellowship.
} 
We note that our theorem is a long-sought cohomological characterization of this particular type of finite $p$-group (which we shall say satisfies the $p C$ condition). Such results are not common in the cohomology of finite groups, as the structure of a group is usually far more rigid than its cohomology. An interesting consequence is that given a group with Cohen-Macaulay cohomology and containing essential elements, the same property will be inherited by any subgroup. Combining the above with a detection theorem due to J. Carlson [C], we obtain

Corollary 2.2. Let $Q$ be a finite group with $G \in S y l_{p}(Q)$; then, if $E \subseteq G \subseteq Q$ is an elementary abelian subgroup of maximal rank, $H^{*}\left(C_{G}(E), \mathbb{F}_{p}\right)$ is CohenMacaulay and has non-trivial essential elements. Moreover, if $H^{*}\left(Q, \mathbb{F}_{p}\right)$ is CohenMacaulay, then it is detected on the cohomology of these centralizers.

Hence we see that in the Cohen-Macaulay situation, there is universal detection by accessible groups with non-trivial essential cohomology; the usefulness of such a result is illustrated for example in the calculations of the mod 2 cohomology of the sporadic simple groups $M_{11}, M_{12}$ and $O^{\prime} N$. We will discuss these examples in $\S 3$, as well as the situation for lower depth, using $M_{22}$ as an example.

We should point out that this paper developed as an attempt to settle problem 4 in J.F. Adams' "Problem Session for Homotopy Theory" held at the 1986 Arcata Topology Conference (see [CCMR], page 438): namely can one give a useful alternative description of $p$-groups with non-zero essential cohomology? M. Feshbach's original conjecture was that $\operatorname{Ess}^{*}(G) \neq 0$ if and only if $G$ satisfies the $p C$ condition (restricted to $p=2$ ). Rusin $[\mathrm{R}]$ provided an example (denoted $\Gamma_{6} a_{2}$ in $[\mathrm{H}])$ of a group of order 32 such that $\operatorname{Ess}^{*}(G) \neq 0$ but without the $2 C$ condition. The extra-special $p$-groups of order $p^{3}$ and exponent $p, p$ an odd prime greater than 3, are counterexamples for $p$ odd (see [L] and $\S 3$ ). In both instances it is apparent that the Cohen-Macaulay condition is absent. However, by a result due to Duflot [D], the $p C$ condition implies Cohen-Macaulay, hence the motivation behind Theorem 2.1.

The main idea in our proof is to exploit the current technical understanding of Cohen-Macaulay cohomology rings, as developed by Benson and Carlson in [BC]. The underlying explicit 'geometric' structure which they develop provides the groundwork for a constructive method used in proving the existence of undetectable cohomology classes given the $p C$ hypothesis.

The paper is organized as follows: in $\S 1$ we provide background in concepts and methods from group cohomology, in $\S 2$ we prove our main results and in $\S 3$ we describe some examples and draw a few conclusions. We are indebted to D. Benson and M. Isaacs for helpful comments.

\section{$\S 1$ Preliminaries}

In this section we will provide the background necessary for the proof of our main results. The books [B] and [AM1] are good general references. We assume throughout (unless otherwise stated) that $G$ is a finite $p$-group and that $k$ is a field of characteristic $p$. To begin we recall 
Definition 1.1. The cohomology ring $H^{*}(G, k)$ is said to be Cohen-Macaulay if there exists a polynomial subalgebra

$$
k\left[\zeta_{1}, \ldots, \zeta_{n}\right] \subseteq H^{*}(G, k)
$$

over which $H^{*}(G, k)$ is a free and finitely generated module.

This is an important condition on the cohomology of a finite group which we shall use decisively in the proof of our main result. We will also need the following basic result due to J. Carlson (see [C], Theorem 2.3)

Theorem 1.2. Let $G$ be a finite group such that $H^{*}(G, k)$ is Cohen-Macaulay. Then its cohomology is detected by restriction to the cohomology of centralizers of elementary abelian subgroups of maximal rank in $G$.

Next we introduce

Definition 1.3. The group $G$ is said to satisfy the $p C$ condition if every element of order $p$ in $G$ is central.

Well known examples of this type of group include abelian $p$-groups and quaternion groups. Less well known is the fact that the 2-Sylow subgroups of the simple groups $P S U_{3}\left(\mathbb{F}_{2^{n}}\right)$ and $S z\left(2^{2 n+1}\right)$ also satisfy this condition.

The two concepts above are related by a result due to J. Duflot [D]

Theorem 1.4. Let $E \subseteq G$ be an elementary abelian subgroup of maximal rank in $G$; then, if $C_{G}(E)$ denotes its centralizer in $G$, the cohomology ring $H^{*}\left(C_{G}(E), k\right)$ is Cohen-Macaulay.

Corollary 1.5. If $G$ satisfies the $p C$ condition, then $H^{*}(G, k)$ is Cohen-Macaulay.

Indeed, if $G$ satisfies the $p C$ condition, then it has a unique central elementary abelian subgroup of maximal rank.

We now introduce another cohomological notion.

Definition 1.6. An element $x \in H^{*}(G, k)$ is said to be essential if it restricts to zero on every proper subgroup in $G$.

If $\Omega(G)$ denotes the collection of all index $p$ subgroups in $G$, then we can consider the map induced by restrictions

$$
H^{*}(G, k) \longrightarrow \bigoplus_{H \in \Omega(G)} H^{*}(H, k) .
$$

The essential elements are precisely those in the kernel of this map. Let us denote the ideal of essential elements by $\operatorname{Ess}^{*}(G) \subset H^{*}(G, k)$. We record without proof some basic properties of this object. 
Proposition 1.7. (1) $\operatorname{Ess}^{*}(G)=0$ if and only if $H^{*}(G, k)$ is detected on proper subgroups. In particular it is zero if $G$ is not a p-group.

(2) $\operatorname{Ess}^{*}(G)$ is an ideal invariant under the action of the Steenrod Algebra.

(3) If $G$ is not elementary abelian, the elements in $\operatorname{Ess}^{*}(G)$ are nilpotent.

(4) If $f: G \rightarrow K$ is a group isomorphism, then it induces a bijection $\operatorname{Ess}^{*}(K) \rightarrow$ $\operatorname{Ess}^{*}(G)$.

It is clear that there exist many interesting groups for which $\operatorname{Ess}^{*}(G)=0$. In fact this vanishing is a key element in most known cohomology calculations (see [AM1] for explicit examples). For example, it has long been understood that detectability on abelian subgroups is a highly desirable situation that arises in a number of important cases. In contrast, a group $G$ with non-zero essential elements in its cohomology contributes new information inherently associated to the group. In turn it will play the part of detector for groups which contain it. Hence it is natural to search for group-theoretic conditions which imply $\operatorname{Ess}^{*}(G) \neq 0$.

\section{$\S 2$ Main Result}

In this section we prove our main result, namely

Theorem 2.1. Let $G$ be a finite $p$-group. Then every element of order $p$ in $G$ is central if and only if $H^{*}(G, k)$ is Cohen-Macaulay and $\operatorname{Ess}^{*}(G) \neq 0$.

Proof. Assume first that every element of order $p$ in $G$ is central. By 1.5, this hypothesis implies that $H^{*}(G, k)$ is Cohen-Macaulay. Hence we may choose elements

$$
\zeta_{1}, \ldots, \zeta_{n} \in H^{*}(G, k)
$$

such that they generate a polynomial subalgebra

$$
k\left[\zeta_{1}, \ldots, \zeta_{n}\right] \subseteq H^{*}(G, k)
$$

over which $H^{*}(G, k)$ is free and finitely generated. Note that $n$ is equal to the $p$-rank of $G$, the rank of the largest elementary abelian subgroup in $G$.

Using theorems 1.3 and 6.3 in $[\mathrm{BC}]$, it follows that we may choose a free generator $z \in H^{N}(G, k)$ for $H^{*}(G, k)$ as a module over $k\left[\zeta_{1}, \ldots, \zeta_{n}\right]$ such that $z=\operatorname{tr}_{E}^{G}(x)$, where $E \subseteq G$ is the central elementary abelian subgroup in $G$ of maximal rank (this element is referred to as the "last survivor").

Now by Quillen's theorem on nilpotent elements (see [AM1]), restriction to $H^{*}(E, k)$ must be injective on $k\left[\zeta_{1}, \ldots, \zeta_{n}\right]$. Let $\zeta_{i}^{\prime}=\operatorname{res}_{E}^{G}\left(\zeta_{i}\right)$, then

$$
k\left[\zeta_{1}^{\prime}, \ldots, \zeta_{n}^{\prime}\right] \subseteq H^{*}(E, k)
$$

is a polynomial subalgebra of maximum Krull dimension $n$. If $\Omega(E)$ denotes the collection of all index $p$ subgroups in $E$, then the map induced by restrictions 


$$
k\left[\zeta_{1}^{\prime}, \ldots, \zeta_{n}^{\prime}\right] \longrightarrow \bigoplus_{E^{\prime} \in \Omega(E)} H^{*}\left(E^{\prime}, k\right)
$$

must have a non-trivial kernel (as can be seen by comparing Krull dimensions). Hence we may choose a non-zero polynomial

$$
p\left(\zeta_{1}^{\prime}, \ldots, \zeta_{n}^{\prime}\right) \in E s s^{*}(E) \cap k\left[\zeta_{1}^{\prime}, \ldots, \zeta_{n}^{\prime}\right] .
$$

We now take the element

$$
p\left(\zeta_{1}^{\prime}, \ldots, \zeta_{n}^{\prime}\right) \cdot x \in H^{*}(E, k)
$$

and apply $t r_{E}^{G}$ to it:

$$
\begin{aligned}
\operatorname{tr}_{E}^{G}\left(p\left(\zeta_{1}^{\prime}, \ldots, \zeta_{n}^{\prime}\right) \cdot x\right) & =\operatorname{tr}_{E}^{G}\left(\operatorname{res}_{E}^{G}\left(p\left(\zeta_{1}, \ldots, \zeta_{n}\right)\right) \cdot x\right) \\
& =p\left(\zeta_{1}, \ldots, \zeta_{n}\right) \cdot \operatorname{tr}_{E}^{G}(x) \\
& =p\left(\zeta_{1}, \ldots, \zeta_{n}\right) \cdot z \neq 0 .
\end{aligned}
$$

We claim that this element is essential. Let $H \subset G$ be any index $p$ subgroup in $G$, then using the double coset formula (see [AM1], II.6), we obtain

$$
\begin{aligned}
\operatorname{res}_{H}^{G}\left[p\left(\zeta_{1}, \ldots, \zeta_{n}\right) \cdot z\right] & =\operatorname{res}_{H}^{G} \operatorname{tr}_{E}^{G}\left[p\left(\zeta_{1}^{\prime}, \ldots, \zeta_{n}^{\prime}\right) \cdot x\right] \\
& =\sum_{[g] \in H \backslash G / E} \operatorname{tr}_{H \cap E^{g}}^{H}\left[g \cdot p\left(\zeta_{1}^{\prime}, \ldots, \zeta_{n}^{\prime}\right) \cdot x\right] .
\end{aligned}
$$

Note however that $E \subseteq G$ is central, hence $E^{g}=E$ and the action in cohomology is trivial. Hence the expression above simplifies to yield

$$
\operatorname{res}_{H}^{G}\left[p\left(\zeta_{1}, \ldots, \zeta_{n}\right) \cdot z\right]=\sum_{[g] \in H \backslash G / E} \operatorname{tr}_{H \cap E}^{H} \operatorname{res}_{H \cap E}^{E}\left[p\left(\zeta_{1}^{\prime}, \ldots, \zeta_{n}^{\prime}\right) \cdot x\right] .
$$

We now consider the two possible cases: either $E \subseteq H$ or not. Assume to begin that $H \cap E \neq E$; in this case we have

$$
r e s_{E \cap H}^{E}\left[p\left(\zeta_{1}^{\prime}, \ldots, \zeta_{n}^{\prime}\right) \cdot x\right] \cdot r e s_{H \cap E}^{E} x=0
$$

as $p\left(\zeta_{1}^{\prime}, \ldots, \zeta_{n}^{\prime}\right) \in H^{*}(E, k)$ is essential by construction. Hence we conclude that

$$
\operatorname{res}_{H}^{G}\left[p\left(\zeta_{1}, \ldots, \zeta_{n}\right) \cdot z\right]=0 .
$$

Now let's assume that $H \cap E=E$; in this case the formula simplifies to

$$
\begin{aligned}
\operatorname{res}_{H}^{G}\left[p\left(\zeta_{1}, \ldots, \zeta_{n}\right) \cdot z\right] & =\sum_{[G: H]} \operatorname{tr}_{H \cap E}^{H}\left[p\left(\zeta_{1}^{\prime}, \ldots, \zeta_{n}^{\prime}\right) \cdot x\right] \\
& =[G: H] \operatorname{tr}_{H \cap E}^{H}\left[p\left(\zeta_{1}^{\prime}, \ldots, \zeta_{n}^{\prime}\right) \cdot x\right]=0 .
\end{aligned}
$$


We conclude that in either case the restriction is zero, and hence $p\left(\zeta_{1}, \ldots, \zeta_{n}\right) \cdot z$ is a non-zero essential element and so $\operatorname{Ess}^{*}(G) \neq 0$.

For the converse we use the following interpretation of 1.2: if there exists an element $\xi \neq 0$ in $H^{*}(G, k)$ such that $\operatorname{res}_{C_{G}(E)}^{G}(\xi)=0$ for all elementary abelian subgroups $E$ of maximal rank with centralizer $C_{G}(E)$, then $H^{*}(G, k)$ is not Cohen-Macaulay.

Hence if $\operatorname{Ess}^{*}(G) \neq 0$ and $H^{*}(G, k)$ is Cohen-Macaulay, we conclude that there exists an elementary abelian subgroup $E \subseteq G$ of maximal rank such that $G=C_{G}(E)$. This clearly implies that all elements of order $p$ in $G$ are central, and therefore our proof is complete.

Remark. Let $G$ be any $p$-group of rank $n$. Then, if $E \subseteq G$ is elementary abelian of this rank, we have shown that $C_{G}(E)$ has essential elements in its cohomology, which in addition is Cohen-Macaulay. If we take $Q$ to be any finite group with $G \in \operatorname{Syl}_{p}(Q)$, then 1.2 will still hold for $Q$. Noting that (up to conjugacy) we may assume $\operatorname{Syl}_{p}\left(C_{Q}(E)\right)=G \cap C_{Q}(E)=C_{G}(E)$, we can deduce

Corollary 2.2. Let $Q$ be a finite group with $G \in S y l_{p}(Q)$; then, if $E \subseteq G \subseteq$ $Q$ is an elementary abelian subgroup of maximal rank, $H^{*}\left(C_{G}(E), k\right)$ is CohenMacaulay and has non-trivial essential elements. Moreover, if $H^{*}(Q, k)$ is CohenMacaulay, then it is detected on the cohomology of these subgroups.

A simple but nevertheless interesting by-product of our theorem is the following

Corollary 2.3. If $H^{*}(G, k)$ is Cohen Macaulay and $\operatorname{Ess}^{*}(G) \neq 0$, then the same will hold for any subgroup of $G$. Similarly if $p$ is an odd prime and $E$ is the maximal elementary abelian subgroup in $G$, the same properties will hold for $G / E$.

Proof. The proof for subgroups follows from the simple observation that the $p C$ condition is inherited on subgroups. Less well known is the fact (pointed out to us by M. Isaacs [I]) that for $p$ an odd prime, if $G$ is $p C$ and $E$ denotes the maximal elementary abelian subgroup, then $G / E$ will also be $p C$. This completes the proof.

We already mentioned in $\S 1$ that from Quillen's work it follows that all the elements in $\operatorname{Ess}^{*}(G)$ are nilpotent provided $G$ is not elementary abelian. The following proposition shows how they appear in the cohomology of groups $Q$ such that $\operatorname{Syl}_{p}(Q)=G$.

Proposition 2.4. Let $G$ be a p-Sylow subgroup of $Q$, then im res $G_{G}^{Q} \cap \operatorname{Ess}^{*}(G)=$ $E_{s s^{*}}(G)^{N_{Q}(G)}$, where $N_{G}(Q)$ denotes the normalizer of $G$ in $Q$. These invariant classes represent elements in the cohomology of $Q$ and in particular the elements in $\operatorname{Ess}^{*}(G)^{\text {Out }(G)}$ are universally stable, i.e. will be in the restriction image for 
any $Q$ with $G$ as a $p$-Sylow subgroup.

Proof. Let $x \in Q$; we begin by observing that the map induced by conjugating with $x$ will induce an isomorphism $c_{x}^{*}: \operatorname{Ess}^{*}(G) \rightarrow \operatorname{Ess}^{*}\left(G^{x}\right)$ (this follows from 1.7).

Now let $Q=\sqcup_{i=1}^{n} G x_{i} G$ be a double coset decomposition. By the CartanEilenberg stability theorem (using the fact that $[Q: G]$ is prime to $p$ ) an element $z \in H^{*}(G, k)$ is in the image of $\operatorname{res}_{G}^{Q}$ if and only if it is stable, i.e.

$$
\operatorname{res}_{G \cap G^{x_{i}}}^{G}(z)=\operatorname{res}_{G \cap G^{x_{i}}}^{G^{x_{i}}} \cdot c_{x_{i}}^{*}(z)
$$

for $i=1,2, \ldots, n$ (see [AM], II.6). If $z \in \operatorname{Ess}^{*}(G)$ and $G^{x_{i}} \neq G$, then both sides are zero and stability follows. If $x_{i} \in N_{Q}(G)$, then the condition simply means that $c_{x_{i}}^{*}(z)=z$ (note that if $\phi \in \operatorname{Aut}(G)$, then by 1.7 it induces a bijection on $\operatorname{Ess}^{*}(G)$ ). We conclude that $\operatorname{Ess}^{*}(G)^{N_{Q}(G)}=\operatorname{imres}_{G}^{Q}$. Finally we note that inner automorphisms act trivially in cohomology and that

$$
\operatorname{Ess}^{*}(G)^{\text {Out }(G)} \subseteq \cap_{Q} \operatorname{Ess}^{*}(G)^{N_{Q}(G)}
$$

where the term on the right is the set of universally stable elements in $\operatorname{Ess}^{*}(G)$.

Remark. In $[\mathrm{MP}]$ it is shown that if $G$ satisfies the $p C$ condition (p odd), then $H^{*}(Q, k) \cong H^{*}(G, k)^{N_{Q}(G)}$ for any $Q$ such that $\operatorname{Syl}_{p}(Q)=G$. For $p=2$, it is possible to show that $H^{*}(G, k) \cong H^{*}\left(N_{Q}(E), k\right)$, where $E \subseteq G$ is the maximal elementary abelian subgroup.

\section{$\S 3$ Examples and conclusions}

The results in this paper indicate that groups of type $p C$ will be a detecting family with non-zero essential cohomology for all groups which satisfy the CohenMacaulay condition on their cohomology. We present a few interesting examples.

Example 3.1. Let $M_{11}$ denote the first Mathieu group. Then its 2-Sylow subgroup is semi-dihedral of order 16, with a unique maximal elementary abelian subgroup of rank 2 which is self-centralizing. $H^{*}\left(M_{11}, \mathbb{F}_{2}\right)$ is Cohen-Macaulay, detected on this subgroup (see [AM1]). Note that in contrast $\operatorname{Syl}_{2}\left(M_{11}\right)$ is not Cohen-Macaulay and is not detected on this elementary abelian subgroup.

Example 3.2. Let $M_{12}$ denote the next Mathieu group; its 2-Sylow subgroup has order 64, a semi-direct product $Q_{8} \times_{T} D_{8}$ and there are three conjugacy 
classes of maximal rank (equal to 3) elementary abelian subgroups. They are all self-centralizing. Hence, as $H^{*}\left(M_{12}, \mathbb{F}_{2}\right)$ is Cohen-Macaulay, they will detect the cohomology (see [AM1]).

Example 3.3. Let $O^{\prime} N$ denote the O'Nan group; its 2-Sylow subgroup has order $2^{9}$, a nonsplit extension $(\mathbb{Z} / 4)^{3} \cdot D_{8}$, and there are exactly 2 conjugacy classes of maximal rank (equal to 3 ) elementary abelian subgroups. One has centralizer $(\mathbb{Z} / 4)^{3}$, the other has centralizer $\mathbb{Z} / 2 \times \mathbb{Z} / 2 \times \mathbb{Z} / 4$. Once again, $H^{*}\left(O^{\prime} N, \mathbb{F}_{2}\right)$ is Cohen-Macaulay and they must detect the cohomology (see [AM2]).

The converse of our main theorem is actually a special consequence of a more general result in $[\mathrm{C}]$. Recall that the depth of $H^{*}(G, k)$ is the longest length of a sequence of regular elements. Then 1.2 extends to the following statement: if depth $H^{*}(G, k) \geq d$, then $H^{*}(G, k)$ is detected on the cohomology of centralizers of elementary abelian subgroups in $G$ of rank $d$. In particular as before we conclude

Proposition 3.4. If $\operatorname{Ess}^{*}(G) \neq 0$, and $H^{*}(G, k)$ has depth $d$, then $G$ centralizes an elementary abelian subgroup of rank $d$.

A plausible next step in characterizing groups with essential cohomology would be to find necessary and sufficient group-theoretic conditions on $G$ so that depth $H^{*}(G, k)=d$, where $d$ is less than the rank of $G$, and $\operatorname{Ess}^{*}(G) \neq 0$.

Examples of such types of groups include the extra-special $p$-groups $G_{p}$ of order $p^{3}$ and exponent $p$, where $p>3$ (for $p=3$ the mod $\mathrm{p}$ cohomology is Cohen-Macaulay and is detected on proper subgroups [MT]). The cohomology $H^{*}\left(G_{p}, \mathbb{F}_{p}\right)$ has depth one and $\operatorname{Ess}^{*}\left(G_{p}\right) \neq 0$ (see [L]). And of course $G_{p}$ does not centralize any rank 2 elementary abelian subgroup. The following examples illustrate a 2-stage detection scheme without the Cohen-Macaulay condition.

Example 3.5. Consider the projective matrix group $L_{3}(4)$; its 2-Sylow subgroup $S$ is of order 64 , a central extension of $(\mathbb{Z} / 2)^{4}$ by $(\mathbb{Z} / 2)^{2}$. An explicit calculation [AM3] shows that $H^{*}(S)$ is not Cohen-Macaulay; however it is detected on subgroups of the form $(\mathbb{Z} / 2)^{4}$ and $Q_{8} \times \mathbb{Z} / 2$, which certainly satisfy the $2 \mathrm{C}$ condition (we are grateful to Jim Milgram for pointing out this detection scheme to us).

Example 3.6. Let $M_{22}$ denote the next Mathieu group after $M_{12}$, its 2-Sylow subgroup has order $2^{7}$, a semi-direct product $(\mathbb{Z} / 2)^{4} \times_{T} D_{8}$. This simple group has 3 conjugacy classes of maximal elementary abelian subgroups: two of rank 4 and one of rank 3 ; they are all self-centralizing. We deduce that $H^{*}\left(M_{22}, \mathbb{F}_{2}\right)$ is not Cohen-Macaulay. The cohomology is detected by its elementary abelian subgroups together with an index two subgroup $R \cong \operatorname{Syl}_{2}\left(L_{3}(4)\right)$ (see [AM4]). Combining this with the previous detection statement, we conclude that $H^{*}\left(M_{22}, \mathbb{F}_{2}\right)$ is detected on elementary abelian subgroups together with subgroups of the form 
$Q_{8} \times \mathbb{Z} / 2$

This last example illustrates how essential cohomology on detecting subgroups can build up cohomology in a complicated group regardless of the Cohen-Macaulay condition.

\section{References}

[AM1] A. Adem and R. J. Milgram, Cohomology of Finite Groups, Springer-Verlag Grundlehren 309 (1994).

[AM2] A. Adem and R. J. Milgram, The subgroup structure and mod 2 cohomology of O'Nan's sporadic simple group, J. Algebra 176 (1995), 288-315.

[AM3] A. Adem and R. J. Milgram, $A_{5}$-invariants, the cohomology of $L_{3}(4)$ and related extensions, Proc. London Math. Soc. (3) 66 (1993), 187-224.

[AM4] A. Adem and R. J. Milgram, The Cohomology of the Mathieu group $M_{22}$, Topology 34(2) (1995), 389-401.

[B] D. Benson, Representations and Cohomology II, Cambridge Studies in Advanced Mathematics 31 (1991), Cambridge University Press.

[BC] D. Benson and J. Carlson, Projective resolutions and Poincaré duality complexes, Tr. AMS 342 (1994), 447-488.

[C] J. Carlson, Depth and transfer maps in the cohomology of groups, Math. Zeit. 218 (1995), 461-468.

[CCMR] G. Carlsson, R. Cohen, H. Miller and D. Ravenel, (Eds.) Algebraic Topology, Lecture Notes in Mathematics 1370 (1989), Springer-Verlag.

[D] J. Duflot, Depth and equivariant cohomology, Comm. Math. Helv. 56 (1981), 627637.

[H] M. Hall and J. Senior, The Groups of Order $2^{n}$, Macmillan, New York 1963.

[I] M. Isaacs, private communication.

[L] I. Leary, The mod p cohomology rings of some p-groups, Math. Proc. Camb. Phil. Soc. 112 (1992), 63-75.

[MP] J. Martino and S. Priddy, The homotopy and cohomology of Swan groups, Math. Zeit. (to appear)

[MT] R. J. Milgram and M. Tezuka, The geometry and cohomology of $M_{12}$ : II, Bol. Soc. Mat. Mex. Vol.1 No.2 (1995), 91-108.

[R] D. Rusin, The cohomology of the groups of order 32, Math. of Comp. Vol. 53 No. 187 (1989), 359-385.

Alejandro Adem

Mathematics Department

University of Wisconsin

Madison, WI 53706 USA

e-mail: adem@math.wisc.edu

(Received: April 18, 1996)
Dikran Karagueuzian

Mathematics Department

University of Wisconsin

Madison, WI 53706 USA

e-mail: dikran@math.wisc.edu 